\title{
Composición química del huevo de Tortuga Golfina Lepidochelys olivacea (Testudines: Cheloniidae) y su potencial como recurso alimenticio
}

\author{
María Isabel Castro-González* \& Fernando Pérez-Gil Romo \\ Instituto Nacional de Ciencias Médicas y Nutrición Salvador Zubirán. Departamento de Nutrición Animal. Vasco de \\ Quiroga 15. Tlalpan. C.P. 14000. México, D.F. México; isacastro55@yahoo.com.mx \\ * Correspondencia
}

Recibido 04-VI-2010. C Corregido 20-II-2011. Aceptado 25-III-2011.

\begin{abstract}
Chemical composition of eggs of the Olive Ridley Lepidochelys olivacea (Testudines: Cheloniidae) and it's potential as a food source. The Olive Ridley is a worldwide distributed species with high nesting production per season, and in La Escobilla Oaxaca, México, there is a 70\% of non-hatched eggs that are lost. In order to evaluate their potential use as a source for human and animal food products, their chemical composition was analyzed. Lyophilized egg samples from 25 turtles were obtained and were analyzed following the analytical methods for fatty acids, protein, fat, ash, moisture, amino acids, vitamins, cholesterol and microbiological agents. The analytical composition obtained was $(\mathrm{g} / 100 \mathrm{~g})$ : moisture (4.7), ash (3.8), protein (53.7), and fat (47.4). The essential amino acid (g aa/100g protein) content was: Ile (4.4), Lys (6.6), Leu (7.4), Met+Cys (8.8), Phe+Tyr (10.8). The vitamin content was: retinol $(340 \mu \mathrm{g} / 100 \mathrm{~g})$, cholecalciferol $(5.9 \mu \mathrm{g} / 100 \mathrm{~g})$ and $8.6 \mathrm{mg} / 100$ tocopherol, $0.3 \mathrm{mg} / 100 \mathrm{~g}$ thiamine and $1.1 \mathrm{mg} / 100 \mathrm{~g}$ riboflavin. The total lipid content (TL), fatty acids (FA), and cholesterol (Chol) were divided into three groups based on the weight of the turtle: (TL) (44.3-48.7-49.1g/100g) and (Chol) (518.4-522.5mg/100g-728.7). A total of 17 Saturated FA (SFA), 8 Monounsaturated FA (MUFA) and 11 Polyunsaturated FA (PUFA) were identified. The most abundant SFA (mg/100g) were: C14:0 (445-772), C16:0 (485-1263); MUFA: C16:1 (456-716), C18:1n-9c (904-1754), and PUFA: C20:4n-6 (105-217); two n-3 fatty acids were identified EPA (48-103) and DHA (97-189). There were significant differences (Fisher, $\mathrm{p}<0.05)$ for: (Chol), total FA, SFA, MUFA, PUFAs and n-3 (EPA + DHA) FA. It was not detected any microbiological agent. In conclusion, lyophilized L. olivacea eggs are an option for its inclusion in the development of food products as they can be used as a high quality biological protein and n-3 fatty acid source for fortification and enrichment. Rev. Biol. Trop. 59 (4): 1729-1742. Epub 2011 December 01.
\end{abstract}

Key words: Lepidochelys olivacea, lyophilized, non-hatched egg, human food, animal feed.

Lepidochelys olivacea (Eschschotlz, 1929), es conocida en español con los nombres de tortuga golfina y oliva y en inglés como Olive Ridley y Pacific Ridley. Es una de las especies de tortuga marina más abundante y con amplia distribución a nivel mundial, principalmente en aguas tropicales y subtropicales de los océanos Indico, Pacífico y Atlántico Central (Richard \& Hughes 1972, Márquez et al. 1996, Abreu 1999, Eckert et al. 2000, Allen 2007).

Esta especie es la única que puede formar arribazones que llegan a reunir más de 100000 hembras con un número de nidadas de tres a cinco por temporada y donde cada hembra deposita en promedio 100 huevos. A nivel mundial, se conocen al menos diez playas en las que actualmente se registran numerosas arribadas de tortuga golfina, las cuales se ubican en México, Costa Rica e India (Richard \& Hughes 1972, Casas 1978, Márquez et al. 1989, 1996).

En México, la fluctuación de la población de tortuga golfina, que anida en las costas del Pacífico, tiende hacia una recuperación sostenida, en especial en el Santuario de La Escobilla en Oaxaca (CONANP 2010a), la cual es una 
de las playas más importantes a nivel mundial para la anidación de esta especie, puesto que se estiman más de un millón de nidos por estación (Ocana 2010). Desde un punto de vista ecológico estas agregaciones han hecho que la tortuga golfina sea vulnerable a los impactos negativos de la anidación masiva, pues estas agregaciones son predecibles en tiempo y espacio. Por otro lado, la densidad de las nidadas juega un papel determinante para la sobrevivencia de la especie, ya que el gran número de ellas en el mismo lugar origina la destrucción de nidos por sobreposición y cambios en las condiciones de incubación, una vez que se ha alcanzado la capacidad de carga en la zona de anidación. También se genera una destrucción de nidos por organismos de la misma especie, de manera que los huevos de las puestas previas son desenterrados por las tortugas de la arribada que le sigue (Albavera 2007, Ocana 2010, Harfush-Melendez 2011, com. pers.). Otros factores biológicos, que afectan la viabilidad de los huevos, están dados por bacterias o por el escarabajo Omorgus suberosus fabricius, cuya presencia se ha reportado por varios años en $\mathrm{La}$ Escobilla, y los cuales destruyen los huevos en las diferentes etapas del desarrollo embrionario (Harfush-Melendez \& López-Reyes 2007).

Por lo tanto, lo anterior ocasiona que de los millones de huevos depositados en una temporada, sólo entre el cinco y el $30 \%$ tenga éxito en el nacimiento de tortugas; en el caso de La Escobilla se ha encontrado que la tasa de eclosión en los últimos años está en el rango del $10 \%$, el resto de los huevos y embriones dan como resultado la acumulación de una gran cantidad de materia orgánica. Este es el principal argumento científico para llevar a cabo una cosecha sustentable de huevo de tortuga, además las comunidades locales pueden hacer uso de esta materia prima como un recurso económico que de otro modo se desperdiciaría (Mrosovsky 2001, Alvabera 2007, CONANP 2009, Ocana 2010, Harfush-Melendez 2011, com. pers.).

Asimismo se describe por primera vez la composición química del huevo de tortuga golfina de La Escobilla con potencial alimenticio, debido a las bajas tasas de eclosión generadas en las zonas seleccionadas por las hembras para desovar, como sugerencia parcial para la creación de una estrategia de aprovechamiento sustentable del recurso y conservación de la especie, de manera que la información aquí presentada es un indicador del tipo y cantidad de nutrimentos de importancia en la alimentación humana y animal.

El huevo de cualquier especie pecuaria es una de las principales fuentes de proteína de alto valor biológico y vitaminas, y si es de origen marino tendrá una mayor concentración de ácidos grasos n-3: ácido eicosapentaenoico (EPA) y ácido docosahexaenoico (DHA), cuyos beneficios han sido demostrados en la salud humana.

El aprovechamiento de los huevos de tortuga golfina que se convierten en material de desecho, dependerá de su valor nutrimental, así como de la estrategia y procedimiento que se desarrolle para su utilización. Una opción podría ser dentro del desarrollo de alimentos para humanos (como un ingrediente rico en proteína de alto valor biológico, ácidos grasos n-3 y vitaminas), y para animales en la industria de los alimentos balaceados (como un ingrediente fortificante, por sus ácidos grasos EPA y DHA). Es por esto que el objetivo del presente trabajo fue determinar la composición química del huevo L. olivacea, como un recurso potencial en alimentación humana y animal.

\section{MATERIAL Y MÉTODOS}

Obtención de las muestras: Las muestras de huevo de tortuga fueron proporcionadas por el Instituto de Ciencias del Mar y Limnología (ICMyL) de la Universidad Nacional Autónoma de México, según el protocolo establecido para la colecta y manejo de huevo de tortuga, establecido por este grupo de trabajo. Los huevos procedían de la zona de anidación llamada La Escobilla, en el Estado de Oaxaca, México, y la colecta se realizó durante la época reproductiva de 2005-2006. De esta manera se nos proporcionaron 250 muestras de huevo liofizado de 25 tortugas (10 huevos por tortuga), las 
cuales se analizaron por triplicado, se presenta la media y desviación estándar de cada análisis.

Análisis químicos: La proteína cruda se analizó en un equipo automatizado Kjeltec 1032 Tecator; la humedad y cenizas se analizaron de acuerdo a las técnicas del A.O.A.C. (2003).

Los lípidos totales y el perfil de ácidos grasos se analizaron de acuerdo a la técnicas adaptadas de Folch et al. (1957), Bligh \& Dyer (1959), Umemura et al. (1993) y Castro et al. (2001) por cromatografía de gases previa extracción con solventes orgánicos. Entonces se utilizó un detector de flama y una columna capilar de 100m. Los ésteres metílicos de los ácidos grasos se identificaron con ayuda de una mezcla de 37 estándares. Los resultados de los ácidos grasos se presentan en $\mathrm{mg} / 100 \mathrm{~g}$ de huevo liofilizado y los análisis se realizaron por triplicado.

Colesterol: La cuantificación se llevó acabo por HPLC de acuerdo a las técnicas descritas por Vicenzo et al. (2005). Aminoácidos: se identificaron y cuantificaron en un cromatógrafo HPLC, Waters AccQ-Tag Chemistry Package (Waters, 1993). Las siguientes vitaminas se analizaron también por HPLC de acuerdo a las técnicas descritas por Keller (1988): retinol, colecalciferol, tocoferol, tiamina y riboflavina.

Los siguientes análisis microbiológicos se llevaron a cabo por duplicado:

1) Bacterias mesófilas aerobias (CU) por vaciado en placa en agar cuenta estándar a $35^{\circ} \mathrm{C}$ por $48 \mathrm{hr}, 2$ ) Hongos y levaduras (PDA) por vaciado en placa en agar papa-dextrosa acidificado e incubado a $25^{\circ} \mathrm{C}$ durante cinco días, 3) Coliformes totales (RVBA) por vaciado en placa en agar bilis rojo violeta y la incubación fue a $35^{\circ} \mathrm{C}$ por $24 \mathrm{~h}$ y 4 ) Salmonella y Shigella sp. (S.S) en tres fases: a) fase de pre-enriquecimiento en caldo lactosa a $35^{\circ} \mathrm{C}$ por $24 \mathrm{~h} \mathrm{~b}$ ) enriquecimiento selectivo en caldo tetrationato y caldo Vassiliadis Rappapport a $35^{\circ} \mathrm{C}$ por $24 \mathrm{~h}$ c) fase de aislamiento en medios selectivos y diferenciales que son XLD (xilosa-lisina-desoxicolato), verde brillante, sulfito de bismuto. Los dos primeros se incuban a $35^{\circ} \mathrm{C}$ por $24 \mathrm{~h}$ y el último a $35^{\circ} \mathrm{C}$ por $48 \mathrm{~h}$. Todas las técnicas se llevaron a cabo de acuerdo a las Normas Oficiales Mexicanas NOM-092, 111, 113 y 114-SSA1-1994 (1994). La preparación de las diluciones de la muestra para la realización de los análisis microbiológicos se llevó a cabo de acuerdo a la Norma Oficial Mexicana NOM110-SSA1-1994(1994), donde se menciona que a partir de muestras sólidas, en este caso huevo liofilizado, se tomaron $10 \mathrm{~g}$ que se llevaron a una dilución primaria y posteriormente a diluciones decimales adicionales con una solución reguladora de fosfatos.

Asimismo, por la importancia que tiene la fracción lipídica para un buen estado de salud de cualquier organismo, se planteó como objetivo secundario determinar si existe variación en la concentración de lípidos totales, ácidos grasos y colesterol, en los huevos procedentes de tortugas anidadoras de diferentes intervalos de peso: Grupo 1 (G1) con ocho tortugas de un peso de 27 a 32kg; Grupo 2 (G2), con ocho tortugas de un peso de 32.1 a $37 \mathrm{~kg}$ y Grupo 3 (G3) con siete tortugas con un peso de $37.1 \mathrm{a} 42.7 \mathrm{~kg}$. (Nota: datos proporcionados por personal del Instituto de Ciencias del Mar y Limnología que realizó el trabajo de campo tanto para la obtención de los huevos y como para el pesaje de las tortugas con el uso de una báscula de piso).

Los resultados de cada nutrimento se sometieron a una prueba de normalidad de Shapiro-Wilk W y un histograma con función de distribución normal, en el que se utilizó el programa de Statistica Statsoft Inc. (2007). Los resultados de lípidos totales, colesterol y ácidos fueron comparados entre tortugas agrupadas en tres grupos de acuerdo a su peso, en donde se manipuló un análisis de varianza de una vía seguido de una prueba de Fisher con un nivel de significancia de $\mathrm{p}<0.05$. Los análisis estadísticos se realizaron en el programa Statistica StatSoft Inc (2007). Los datos en los Cuadros se expresan como media \pm DE.

\section{RESULTADOS}

En el Cuadro 1 se presenta la composición química del huevo de L. olivacea liofilizado y como referencia el huevo deshidratado de 


\section{CUADRO 1}

Composición química de huevo liofilizado-no eclosionado de tortuga golfina Lepidochelys olivacea y de huevo deshidratado de gallina (Gallus domesticus)

TABLE 1

Chemical composition of lyophilized-non hatched egg of olive ridley (Lepidochelys olivacea) and dehydrated laying hen egg (Gallus domesticus)

\begin{tabular}{lcc}
\multicolumn{1}{c}{ Nutrimento } & Lepidochelys olivacea & Gallus domesticus $^{1,2}$ \\
Humedad g/100g & $4.67 \pm 0.004$ & $2.8-5$ \\
Proteína $\mathrm{g} / 100 \mathrm{~g}$ & $53.69 \pm 0.05$ & $46-49$ \\
Grasa g/100g & $47.40 \pm 4.0$ & $37-41$ \\
Cenizas g/100g & $3.78 \pm 0.007$ & $3.5-3.65$ \\
Retinol $\mu \mathrm{g} / 100 \mathrm{~g}$ & $340.2 \pm 0.03$ & 825 \\
Colecalciferol $\mu \mathrm{g} / 100 \mathrm{~g}$ & $5.91 \pm 0.21$ & 5 \\
Tocoferol $\mathrm{mg} / 100 \mathrm{~g}$ & $8.61 \pm 0.4$ & 5.8 \\
Tiamina $\mathrm{mg} / 100 \mathrm{~g}$ & $0.32 \pm 0.08$ & 0.19 \\
Riboflavina $\mathrm{mg} / 100 \mathrm{~g}$ & $1.15 \pm 0.01$ & 1.54 \\
\hline
\end{tabular}

1,2 Souci et al. 2000, Sotelo \& González 2000.

gallina (Gallus domesticus), por ser el huevo de esta especie el de mayor consumo. En el Cuadro 2 se presenta el contenido de aminoácidos del huevo en estudio en comparación con el contenido de aminoácidos presentes en huevo de gallina deshidratado y aminoácidos de harina de pescado.

En el Cuadro 3 se presenta el contenido de lípidos totales (LT), colesterol y el perfil de ácidos grasos en huevos liofilizados de L. olivacea de acuerdo con el peso de las tortugas de los tres grupos. La concentración de lípidos totales (g/100g) varió de 44.3 a 49.1, pero no se observó diferencia significativa $\mathrm{p}>0.05$ entre grupos.

El contenido de colesterol $(\mathrm{mg} / 100 \mathrm{~g})$ presentó valores de 518.4 (G1) a 728.7 (G3); se identificaron 36 ácidos grasos (AG), de los cuales 17 fueron AG saturados, 8 AG monoinsaturados y $11 \mathrm{AG}$ poliinsaturados. En el grupo 3 (G3), se dio una mayor concentración en 27 AG en comparación con G1 y G2. Asimismo, se identificaron cuatro ácidos grasos $\mathrm{n}-3$ : C18:3n-3, C20:3n-3, C20:5n-3 y C22:6n-3; cuatro ácidos grasos n-6: C18:2n-6, C18:3n-6,
C20:3n6 y C20:4n-6; y dos ácidos grasos trans: C18:1n-9t y C18:2n-6t.

Los AG más abundantes en los tres grupos de peso fueron los: C14:0, C16:0, C16:1, C18:1n-9c, C20:4n-6 conocido como ácido araquidónico (AA), C20:5n-3 conocido por sus siglas en inglés como (EPA) y C22:6n-3 conocido como (DHA). La concentración total de todos éstos ácidos grasos fue mayor en el G3, en comparación con los demás grupos.

Al analizar los datos de cada tortuga se observó que la tortuga con el menor peso $(27.6 \mathrm{~kg})$ presentó la mayor concentración de ácidos grasos (AGT=ácidos grasos totales) con $10685 \mathrm{mg} / 100 \mathrm{~g}$, en comparación con las otras tortugas de su grupo (G1), en donde se observaron, por ejemplo, valores de $2163 \mathrm{mg} / 100 \mathrm{~g}$ en un ejemplar de $30.9 \mathrm{~kg}$. Lo mismo ocurrió para la sumatoria de los ácidos grasos saturados y monoinsaturados 5387 y 4263 $\mathrm{mg} / 100 \mathrm{~g}$, respectivamente, contra 702 y 1154 $\mathrm{mg} / 100 \mathrm{~g}$ presentes en otras tortugas del G1; así como para el total de los ácidos grasos n-3 (426mg/100g) contra $107 \mathrm{mg} / 100 \mathrm{~g}$ observados 
CUADRO 2

Contenido de aminoácidos en huevo liofilizado-no eclosionado de tortuga golfina L. olivacea, en huevo de gallina deshidratado y en dos tipos de harina de pescado

TABLE 2

Amino acids in lyophilized-non hatched L. olivacea egg dehydrated laying hen egg and two different fish meals

\begin{tabular}{lccc}
\multicolumn{1}{c}{ Aminoácido $\mathrm{g} / 100 \mathrm{~g}$ de proteína } & L. olivacea & Gallus domesticus $^{1,2}$ & Harinas de pescado $^{3,4}$ \\
Isoleucina & $4.42 \pm 0.12$ & $2.58-3.22$ & $2.8-5.7$ \\
Leucina & $7.40 \pm 0.24$ & $4.15-4.50$ & $5.0-8$ \\
Lisina & $6.62 \pm 0.16$ & $3.25-3.37$ & $5.2-6.5$ \\
Metionina & $5.55 \pm 0.21$ & $1.53-1.64$ & $2.2-2.3$ \\
Cistina & $3.23 \pm 0.09$ & $1.15-1.98$ & NR \\
Fenilalanina & $6.10 \pm 0.24$ & $2.64-2.70$ & $3.1-4.9$ \\
Tirosina & $4.72 \pm 0.21$ & $1.91-2.08$ & $2.2-3.1$ \\
Treonina & $6.48 \pm 0.16$ & $2.12-2.60$ & $2.3-2.8$ \\
Valina & $6.53 \pm 0.28$ & $3.18-3.9$ & $3.2-6.8$ \\
Arginina & $6.45 \pm 0.12$ & $3.01-3.13$ & $4.6-6.6$ \\
Histidina & $3.02 \pm 16$ & $1.14-1.19$ & $2.3-3.5$ \\
Alanina & $5.45 \pm 0.21$ & $2.63-3.21$ & NR-7.5 \\
Asparagina & $8.32 \pm 0.16$ & $4.65-5.29$ & NR-9.2 \\
Glutamina & $10.87 \pm 0.19$ & $5.92-6.39$ & NR-16.6 \\
Glicina & $4.07 \pm 0.09$ & $1.54-1.89$ & NR-11.5 \\
Prolina & $3.68 \pm 0.16$ & $1.57-2.09$ & NR \\
Serina & $6.20 \pm 0.24$ & $2.79-4.08$ & NR-4.6 \\
\hline
\end{tabular}

${ }^{1,2}$ Souci et al. 2000, Sotelo y González 2000; ${ }^{3,4}$ Hernández et al. 2008, Hu et al. 2008; NR=no reportado.

en la misma tortuga de casi $31 \mathrm{~kg}$ del G1. En cuanto a los ácidos grasos poliinsaturados totales este ejemplar presentó el segundo valor más alto $(1033 \mathrm{mg} / 100 \mathrm{~g})$, siendo el mayor de $1130 \mathrm{mg} / 100 \mathrm{~g}$.

La tortuga con el mayor peso $(42.7 \mathrm{~kg})$, presentó diferencias en cuanto al porcentaje de sus ácidos grasos en comparación con las otras tortugas de su grupo (G3): AGS 53.5\%, AGM $28.8 \%$ y AGP $17.7 \%$, contra $38.3 \%$, $50.2 \%$ y $9.7 \%$, respectivamente. Los AG n-3 de este ejemplar fueron semejantes a la media del grupo (313.8 mg/100g de AGn-3 totales).

De esta forma se observó diferencia significativa Fisher, $\mathrm{p}<0.05$, entre grupos de peso, para el contenido de los ácidos grasos n-3 $\mathrm{EPA}+\mathrm{DHA}$, en donde G1 y G3 son mayores a G2. En la Fig. 1, se presenta el contenido de los ácidos grasos agrupados por tipología en: ácidos grasos saturados (AGS), ácidos grasos monoinsaturados (AGM) y ácidos grasos poliinsaturados (API), conjuntados a su vez de acuerdo a los diferentes grupos de peso de las tortugas Independientemente del peso se notó que los AGP de todos los grupos presentaron en una concentración menor; mientras que los AGS y AGM tuvieron una mayor concentración.

De manera importante se hace notar que en los análisis microbiológicos no se detectó la presencia de microorganismos patógenos en la harina de huevo liofilizado de L. olivacea .

\section{DISCUSIÓN}

Como recurso potencial en alimentación humana. En el huevo de tortuga analizado, la fracción más abundante fue la proteína con una 
CUADRO 3

Lípidos totales, colesterol y ácidos grasos en huevos liofilizados-no eclosionados de tortugas marinas L. olivacea agrupadas por peso

TABLE 3

Total lipids, cholesterol and fatty acids in lyophilized-non hatched eggs of L. olivacea grouped by weight

\begin{tabular}{|c|c|c|c|}
\hline & Grupo 1 & $32.1-37 \mathrm{~kg}$ & $37.1-42.7 \mathrm{~kg}$ \\
\hline Lípidos Tot. (g/100g) & $48.74 \pm 12^{\mathrm{a}}$ & $49.11 \pm 6^{\mathrm{a}}$ & $44.33 \pm 10^{\mathrm{a}}$ \\
\hline Colesterol (mg/100g) & $518.4 \pm 97^{\mathrm{a}}$ & $522.5 \pm 2^{\mathrm{a}}$ & $728.7 \pm 211^{\mathrm{b}}$ \\
\hline \multicolumn{4}{|l|}{ AG (mg/100g) } \\
\hline $\mathrm{C} 4: 0$ & $36.92 \pm 76$ & $67.81 \pm 87$ & $46.91 \pm 84$ \\
\hline C6:0 & $5.02 \pm 9$ & $25.6 \pm 42$ & $33.37 \pm 48$ \\
\hline $\mathrm{C} 8: 0$ & $3.45 \pm 5$ & $0.72 \pm 0.3$ & $3.13 \pm 4$ \\
\hline $\mathrm{C} 10: 0$ & $3.88 \pm 7$ & $0.55 \pm 0.4$ & $38.85 \pm 75$ \\
\hline C11:0 & $1.16 \pm 1$ & $0.86 \pm 0.5$ & $1.11 \pm 1.3$ \\
\hline $\mathrm{C} 12: 0^{\star}$ & $22.08 \pm 14^{\mathrm{a}}$ & $12.53 \pm 8^{\mathrm{ab}}$ & $55.97 \pm 83^{\mathrm{ac}}$ \\
\hline $\mathrm{C} 13: 0$ & $3.13 \pm 1.4$ & $2.00 \pm 1.4$ & $111.47 \pm 283$ \\
\hline $\mathrm{C} 14: 0^{*}$ & $742.96 \pm 351^{\mathrm{a}}$ & $445.11 \pm 259^{\mathrm{a}}$ & $772.21 \pm 504^{\mathrm{a}}$ \\
\hline $\mathrm{C} 15: 0$ & $57.49 \pm 22$ & $92.48 \pm 196$ & $76.93 \pm 35$ \\
\hline $\mathrm{C} 15: 1$ & $21.44 \pm 33$ & $14.64 \pm 29$ & $22.26 \pm 33$ \\
\hline $\mathrm{C} 16: 0^{*}$ & $912.01 \pm 365^{\mathrm{a}}$ & $485.33 \pm 295^{\mathrm{ab}}$ & $1263.65 \pm 972^{\mathrm{ac}}$ \\
\hline $\mathrm{C} 16: 1^{*}$ & $650.07 \pm 271^{\mathrm{a}}$ & $456.62 \pm 326^{\mathrm{a}}$ & $716.57 \pm 301^{\mathrm{a}}$ \\
\hline $\mathrm{C} 17: 0$ & $66.25 \pm 24$ & $119.57 \pm 280$ & $83.19 \pm 37$ \\
\hline $\mathrm{C} 17: 1$ & $110.15 \pm 37$ & $65.02 \pm 30$ & $126.92 \pm 43$ \\
\hline C18:0* & $246.04 \pm 88^{\mathrm{a}}$ & $146.23 \pm 78^{\mathrm{ab}}$ & $331.88 \pm 257^{\mathrm{ac}}$ \\
\hline $\mathrm{C} 18: \ln 9 \mathrm{t}^{*}$ & $46.66 \pm 52^{\mathrm{a}}$ & $50.29 \pm 87^{\mathrm{a}}$ & $72.97 \pm 85^{\mathrm{a}}$ \\
\hline $\mathrm{C} 18: \ln 9 \mathrm{c}^{*}$ & $1741.49 \pm 630^{\mathrm{a}}$ & $904.29 \pm 552^{\mathrm{b}}$ & $1754.22 \pm 1110^{\mathrm{ac}}$ \\
\hline $\mathrm{C} 18: 2 \mathrm{n} 6 \mathrm{t}$ & $7.64 \pm 3$ & $229.06 \pm 747$ & $9.17 \pm 5$ \\
\hline $\mathrm{C} 18: 2 \mathrm{n} 6 \mathrm{c}^{*}$ & $108.57 \pm 33^{\mathrm{a}}$ & $57.86 \pm 30^{\mathrm{b}}$ & $147.05 \pm 94^{\mathrm{ac}}$ \\
\hline $\mathrm{C} 18: 3 \mathrm{n} 6$ & $38.08 \pm 50$ & $33.12 \pm 45$ & $56.28 \pm 64$ \\
\hline $\mathrm{C} 18: 3 \mathrm{n} 3^{*}$ & $19.33 \pm 6^{\mathrm{a}}$ & $12.22 \pm 6^{\mathrm{ab}}$ & $26.59 \pm 16^{\mathrm{ac}}$ \\
\hline C20:0 & $30.53 \pm 36$ & $18 \pm 23$ & $48.96 \pm 51$ \\
\hline C20:1 & $56.81 \pm 19$ & $35.32 \pm 21$ & $65.59 \pm 23$ \\
\hline $\mathrm{C} 20: 2 \mathrm{n} 9$ & $15.09 \pm 6$ & $16.59 \pm 29$ & $18.06 \pm 9$ \\
\hline C20:3n 3 & $26.26 \pm 24$ & $20.04 \pm 22$ & $40.43 \pm 36$ \\
\hline C20:3n6 & $10.44 \pm 4$ & $7.31 \pm 6$ & $13.02 \pm 6$ \\
\hline $\mathrm{C} 20: 4 \mathrm{n} 6^{*}$ & $204.48 \pm 82^{\mathrm{a}}$ & $105.66 \pm 63^{\mathrm{b}}$ & $216.90 \pm 78^{\mathrm{ac}}$ \\
\hline C21:0 & $4.11 \pm 4$ & $24.56 \pm 64$ & $6.17 \pm 10$ \\
\hline C22:0 & $21.79 \pm 18$ & $18.02 \pm 21$ & $34.79 \pm 31$ \\
\hline $\mathrm{C} 20: 5 \mathrm{n} 3^{*}$ & $88.79 \pm 34^{\mathrm{a}}$ & $48.32 \pm 25^{\mathrm{b}}$ & $103.34 \pm 51^{\mathrm{ac}}$ \\
\hline $\mathrm{C} 22: \ln 13$ & $13.14 \pm 4$ & $13.96 \pm 31$ & $6.65 \pm 3$ \\
\hline C22:2 & $16.34 \pm 14$ & $14.30 \pm 17$ & $26.90 \pm 26$ \\
\hline C23:0 & $544.66 \pm 939$ & $4.19 \pm 7$ & $0.04 \pm 0$ \\
\hline C24:0 & $11.29 \pm 5$ & $1.32 \pm 0.7$ & $17.91 \pm 35$ \\
\hline $\mathrm{C} 24: \ln 9$ & $93.38 \pm 34$ & $57.44 \pm 29$ & $104.57 \pm 40$ \\
\hline $\mathrm{C} 22: 6 \mathrm{n} 3^{*}$ & $159.02 \pm 71^{\mathrm{a}}$ & $97.22 \pm 60^{\mathrm{ab}}$ & $189.60 \pm 100^{\mathrm{ac}}$ \\
\hline
\end{tabular}

* Ácidos grasos de importancia nutricional en alimentación humana Por columnas, ${ }^{a}, \mathrm{~b}, \mathrm{c}$ literales distintas indican diferencia significativa entre grupos $(\mathrm{p}<0.05)$. 


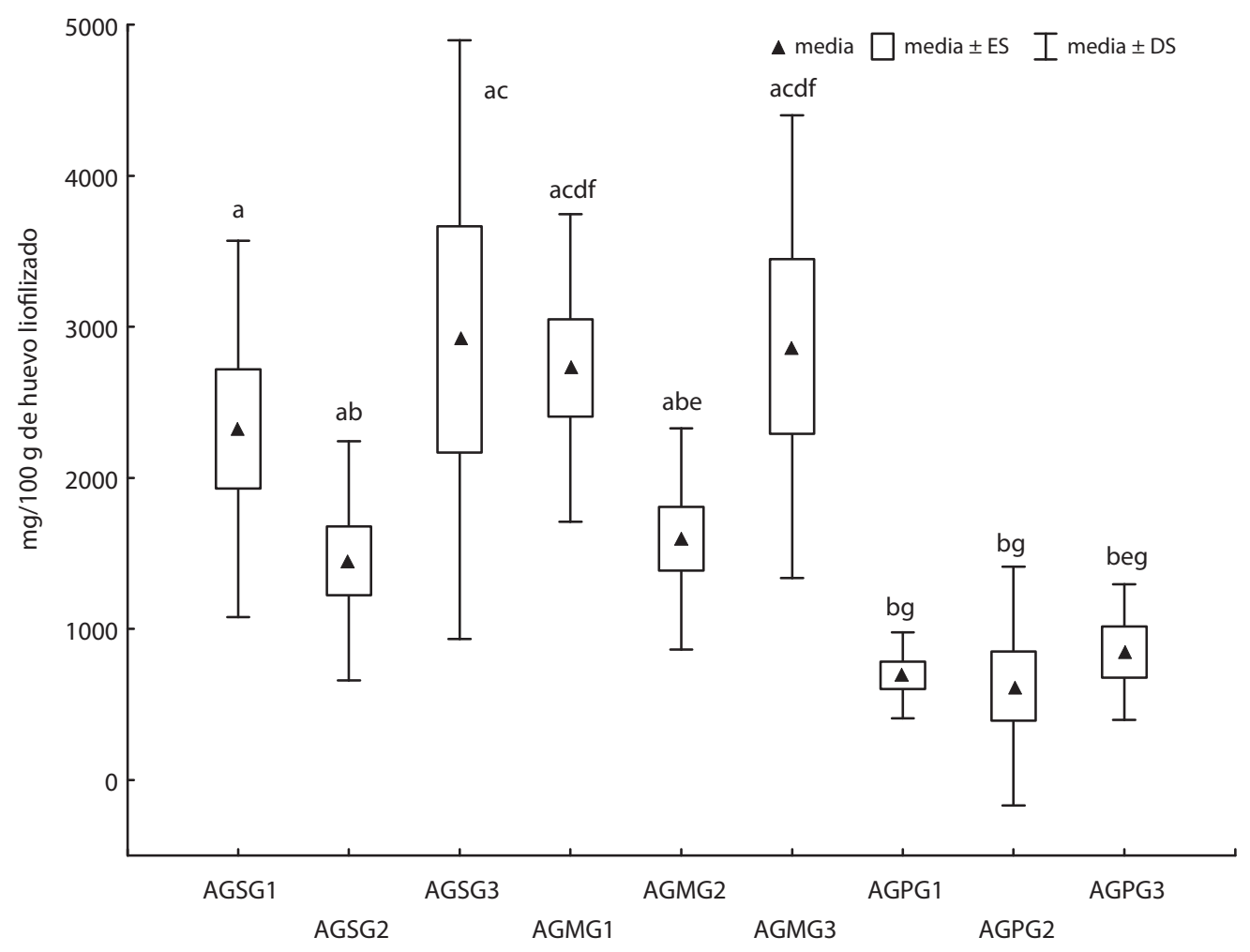

Fig. 1. Ácidos grasos saturados (AGS), monoinsaturados (AGM), poliinsaturados (AGPI) y poliinsaturados n-3 (AGPIn-3) en huevos liofilizados de L. olivacea por grupos de peso. Grupo 1=27-32kg; Grupo 2=32.1-37kg; Grupo 3=37.1-42.7kg • a, b, c, d, e, f, g letras distintas indican diferencia significativa entre grupos (Fisher, $\mathrm{p}<0.05$ ).

Fig. 1. Saturated fatty acids (AGS), monounsaturated fatty acids (AGM), polyunsaturated fatty acids (AGPI) and polyunsaturated n-3 fatty acids (AGPIn-3) presents in lyophilized eggs of L. olivacea by weigh group. Group 1=27-32kg; Group 2=32.1-37kg; Group 3=37.1-42.7kg $\bullet \mathrm{a}, \mathrm{b}, \mathrm{c}, \mathrm{d}, \mathrm{e}, \mathrm{f}, \mathrm{g}$ different letters indicate significant difference between groups (Fisher, $\mathrm{p}<0.05)$.

concentración mayor a la del huevo de gallina, lo mismo ocurrió para los lípidos totales (grasa); mientras que las cenizas que representan el contenido de los minerales, fueron semejantes en ambas especies. De acuerdo a la Norma mexicana NMX-F330S-S-1979, el valor máximo permitido de humedad y cenizas para el huevo entero de gallina deshidratado es de 5 y $4 \%$ respectivamente; el huevo deshidratado de L. olivacea presento $4.7 \%$ y $3.8 \%$, de estos nutrimentos.

Las ingestas diarias recomendadas (IDR) de los nutrimentos han sido emitidas por organismos internacionales como FAO, OMS, UNU, INCAP (Bourges et al. 2005). La IDR para proteína en adultos es de $0.75 \mathrm{~g} / \mathrm{kg}$ y para niños y adolescentes de 0.88 a $1.95 \mathrm{~g} / \mathrm{kg}$, respectivamente (Bourges et al. 2009). Con base en estos datos se estima que para una persona de $60 \mathrm{~kg}$, el consumo de $20 \mathrm{~g}$ harina liofilizada de tortuga golfina, aportaría 33\% de la IDR de proteína.

El huevo liofilizado de tortuga marina presentó una concentración de colesterol muy baja (518.4-728.7mg/100g), en comparación con lo reportado por Sotelo y González (2000) y Souci et al. (2000) en el huevo de gallina deshidratado (1 498 a 2 162mg/100g). Estos resultados corroboran lo encontrado por Mora-Castro et al. (1997), en huevo fresco de 
tortuga golfina donde el contenido de colesterol fue $50 \%$ menor en comparación con huevo fresco de gallina. Entre el colesterol del grupo de mayor peso (G3) y el de los otros grupos se detectó diferencia significativa (Fisher, $\mathrm{p}<0.05$ ).

Aunque, el huevo de tortuga contiene menor concentración de colesterol respecto al huevo de gallina, los valores aquí presentados corresponden a más del doble de los valores diarios de colesterol recomendados para humano. Sin embargo, estas concentraciones de colesterol no afectarían la salud humana siempre y cuando la harina de huevo de tortuga se use en cantidades menores a $100 \mathrm{~g}$, y dentro de los límites establecidos para el huevo de gallina, especialmente en personas con problemas cardiovasculares. La IDR para colesterol en adultos es de <200mg (Bourges et al. 2009), por lo que incluir $20 \mathrm{~g}$ de harina liofilizada de L. olivacea aportaría entre 51.8 y $72.8 \%$ de lo recomendado.

En las vitaminas liposolubles analizadas se observó en el huevo de tortuga menos de la mitad de la concentración de retinol (vitamina A) en comparación con el huevo de gallina deshidratada. Debido a que las recomendaciones para adultos son de $900 \mu \mathrm{g} /$ día para hombres y $700 \mu \mathrm{g} /$ día para mujeres, el consumo de $20 \mathrm{~g}$ de huevo liofilizado de tortuga golfina aportaría casi el $10 \%$ de la cantidad recomendada. La concentración de colecalciferol (vitamina D) fue similar entre ambas harinas (5.9 y $5 \mu \mathrm{g} / 100 \mathrm{~g}$, tortuga y gallina respectivamente), y mayor para el tocoferol (vitamina E) (8.6 y $5.8 \mathrm{mg} / 100 \mathrm{~g}$ ). La recomendación de vitamina D y vitamina E para adultos es de $5 \mu \mathrm{g}$ y $15 \mathrm{mg} /$ día (Bourges et al. 2009).

La concentración de tiamina fue mayor en el huevo de tortuga en comparación con el de gallina $(0.32$ y $0.19 \mathrm{mg} / 100 \mathrm{~g})$, mientras que la riboflavina presenta valores semejantes entre ambas especies $(1.15$ y $1.54 \mathrm{mg} / 100 \mathrm{~g}$, respectivamente). La IDR es de: 1.0 y $0.9 \mathrm{mg}$ de tiamina y 1.30 y $1.10 \mathrm{mg}$ de riboflavina, en hombres y mujeres adultos, respectivamente (Wardlaw et al. 2004, Bourges et al. 2009). Por lo que el consumo de $20 \mathrm{~g}$ de harina de $L$. olivacea aportaría el $6.4 \%$ de tiamina y $17.7 \%$ de riboflavina de la IDR.

Los aminoácidos mas abundantes en la tortuga fueron (g/100g): Glu (10.87), Asp (8.32) y Leu (7.40), mientras que la menor concentración se presentó en His (3.02), Cis (3.23), Pro (3.68), Gli (4.07) e Ile (4.42), el resto de los aminoácidos se presentaron en valores alrededor de seis. En comparación con el huevo de gallina deshidratado, los aminoácidos de huevo liofilizado de L. olivacea presentaron una mayor concentración, los cuales incluían a los aminoácidos esenciales, de esta forma se sitúa como un ingrediente con alto potencial proteínico de alto valor biológico. La recomendación de FAO/OMS/UNU es de: Ile (1.3), Leu (1.9), Lis (1.6), Tre (0.9), Trp (0.5),Val (1.3), para los azufrados (1.7) y aromáticos (1.9) g/100g (Bourges et al. 2009).

En relación con el contenido de ácidos grasos se percibió que el AG saturado más abundante en los tres grupos es el C16:0 (485.3-1 $263.6 \mathrm{mg} / 100 \mathrm{~g}$ ) y el menos abundante fue el C11:0 (0.86-1.1 mg/100g). El ácido graso más abundante de todos los ácidos grasos identificados fue el 18:1n9c, con valores de 904.3 a $1754.2 \mathrm{mg} / 100 \mathrm{~g}$, el cual también fue el más abundante en yema de huevo de otras especies de tortugas, según lo informado por Stephenson \& Owens (1999) para Chelonia mydas y Trachemys scripta. El segundo AG monoinsaturado con concentración elevada fue C16:1 con valores de 456.6 a $716.6 \mathrm{mg} / 100 \mathrm{~g}$.

El ácido graso poliinsaturado más abundante fue el C20:4n-6 (105.7-216.9mg/100g). Los ácidos grasos poliinsaturados del grupo C18 se han reportado como esenciales en reptiles, puesto que no pueden ser sintetizados y deben obtenerse de la dieta (Stephenson \& Owens 1999). Los encontrados en el huevo de L. olivacea fueron C18:2n6t, C18:2n6c, $\mathrm{C} 18: 3 \mathrm{n} 6$ y C18:3n3. No se encontraron reportes sobre la importancia nutrimental de otros ácidos grasos en tortugas marinas, sin embargo, en la alimentación humana un buen número de ácidos grasos son importantes en la dieta, y se detectaron en la mayoría de ellos diferencia significativa (Fisher, $\mathrm{p}<0.05$ ) entre los 
diferentes grupos de peso. Algunos autores sugieren que la dieta de los animales puede afectar directamente la composición de la yema y que su perfil lipídico está determinado por una combinación entre el consumo dietético materno y el metabolismo (Stephenson \& Owens 1999, Craven et al. 2008).

Con lo cual se analizó estadísticamente el contenido de ácidos grasos totales, dados por la sumatoria de los ácidos grasos saturados (AGS), ácidos grasos monoinsaturados (AGM) y ácidos grasos poliinsaturados (AGP). Entre las tortugas del G2 y G3 se encontró diferencia significativa $(\mathrm{p}<0.05)$. Austin et al. (2008) determinaron en huevo de gallina deshidratado un contenido de $37.6 \%$ de AGS, $45.3 \%$ de AGM y $17.1 \%$ de AGP, a diferencia de lo observado en el presente estudio: mayor concentración de AGS y menor de AGP. Al comparar entre los tipos de ácidos grasos y los grupos de peso, se halló diferencia significativa (Fisher, $\mathrm{p}<0.05$ ), con una tendencia a disminuir la concentración de AGP en los tres grupos de tortugas en comparación con la cantidad observada para los otros tipos de ácidos grasos. Con las tortugas de grupo 2 se obtuvo menor concentración para los tres grupos de ácidos grasos.

Así bien, se observaron diferencias significativas (Fisher, $\mathrm{p}<0.05$ ) en los lípidos totales (los cuales son una estimación general de la fracción grasa de un alimento dada por colesterol, esteroles, ácidos grasos, vitaminas liposolubles, fosfolípidos, pigmentos y demás lípidos), estos resultados nos sugieren dos teorías: 1) que conforme la tortuga crece, a partir de un punto dado, su alimentación se vuelve mas variada por una posible área de alimentación más extensa y por lo tanto la cantidad de ácidos grasos totales se eleva; o 2) que a partir de cierta edad (aludida al peso) ocurre un cambio en el metabolismo de las tortugas, que ocasiona un mayor almacenamiento de los ácidos grasos que son posteriormente depositados el huevo durante su formación. Los lípidos de la yema de los huevos de tortuga proporcionan nutrimentos vitales a los embriones para el desarrollo y sobrevivencia de las crías (Stephenson \& Owens 1999); no obstante, se desconoce el papel metabólico y fisiológico que ejercen los ácidos grasos n-3 en las tortugas marinas.

Los ácidos grasos n-3 son de suma importancia en la salud humana, por sus efectos cardioprotectores y antiinflamatorios, principalmente los ácidos grasos conocidos por sus siglas en inglés como EPA (C20:5n-3) y DHA (C22:6n-3). La concentración de ácidos grasos (EPA+DHA) y sus diferencias estadísticas entre grupos sugieren que en tortugas de peso medio (entre 32 y $37 \mathrm{~kg}$ ) agrupadas en G2, existe una utilización diferencial de los ácidos grasos n-3, pues éstos no son depositados en los huevos durante el período en el que se forma el huevo, mientras que en las tortugas de mayor peso se observó una mayor deposición de éstos ácidos grasos de la progenitora al embrión.

La cantidad de ácidos grasos n-3 obtenida, independientemente de los pesos de las tortugas, nos indica el elevado potencial que existe en esta materia prima desperdiciada, ya que los valores encontrados de EPA y DHA (48.3-103.3 y $97.2-189.6 \mathrm{mg} / 100 \mathrm{~g}$, respectivamente) proponen al huevo de tortuga como un recurso potencial rico en n-3. Estos valores son semejantes a los de algunos pescados de consumo humano, tales como (mg/100 EPA y DHA): merluza (Merluccius productos) (22.5 y 102), jurel (Caranx hippos) (4.0 y 117.7), cabrilla (Mycteroperca xenarcha) (13.9 y 85.1) y mayores a otras especies comestibles: cazón (Carcharhinus porosus) (1.5 y 20.7), bagre marino (Bagre panamensis) (3.8 y 35.7), lengua de williamsi (Symphurus williamsi) (9.1 y 31.2) (Castro-González \& Miranda-Becerra 2009, Castro-González \& Miranda 2010, Castro-González 2010).

El huevo de tortuga liofilizado no desarrolló ningún microorganismo patógeno, por lo que la harina de L. olivacea elaborada de acuerdo a la técnica de Calderón et al (2008), cumple con las especificaciones para huevo deshidratado o en polvo para ser consumido por humanos (NMX-F-330-S 1979, NOM159 1996). El liofilizado es una técnica de conservación efectiva, sin embargo, un estudio de Mora-Castro et al. (1997) sobre la calidad física y microbiológica 
de huevo fresco de esta misma especie indicó que ocurre un crecimiento logarítmico de algunos patógenos a partir del día 11 de su recolecta a temperatura ambiente. Los análisis de Salmonella en ese estudio, fueron negativos a este tiempo y los huevos refrigerados presentaron una buena calidad microbiológica después de dos meses. Esta información es importante, si se considera que para desarrollar harina de $L$. olivacea pudiera ser necesario, después de ser recolectados, transportar los huevos al lugar donde se estableciese una planta harinera.

En la alimentación animal, la harina de pescado es comúnmente empleada, pues proporciona una fuente concentrada de proteína de alto valor biológico y ácidos grasos n-3 (EPA y DHA), así como vitaminas y minerales, principalmente fósforo disponible para los animales y vitaminas del complejo B, vitamina A y D (Rice 1999). Los grupos pecuarios en donde se emplea con éxito la harina de pescado son: aves de corral, aves ponedoras, cerdos y rumiantes (vacas lecheras, ganado vacuno y ovinos) (IFOMA 2009). En acuacultura (camarones y peces), también se utiliza harina de: pescado, algas marinas, carne porcina, sangre, desperdicios avícolas, vísceras de atún, subproductos de animales de rastro, recicladas de origen animal, etc. (Cruz-Suárez et al. 2000, Hernández et al. 2004, 2008, Carrillo et al. 2008, Akegbejo \& Fasakin 2008).

La composición química de las harinas de pescados presentan un contenido de proteína cruda de 60-70.1g/100g, el contenido lipídico es de $2-9.3 \mathrm{~g} / 100 \mathrm{~g}$, cenizas $3.8-12.8 \mathrm{~g} / 100 \mathrm{~g}$. Al comparar estos valores con la harina de L. olivacea, el contenido de proteína de esta última fue menor en un $10 \%$, mientras que el contenido lipídico fue aproximadamente siete veces mayor al promedio de grasas reportado para harinas de pescado (Hérnández et al. 2008, Hu et al. 2008). Los valores de cenizas de la harina de tortuga fueron iguales al valor mínimo informado para harinas de pescado (Hérnández et al. 2008, Hu et al. 2008). El huevo de L. olivacea tiene un contenido elevado de tocoferol comparando con las harinas de pescado (8.6 y $0.5 \mathrm{mg} / 100 \mathrm{~g}$, respectivamente), lo mismo ocurre para tiamina 0.32 y $0.07 \mathrm{mg} / 100 \mathrm{~g}$, y para riboflavina: 1.15 y $0.48 \mathrm{mg} / 100 \mathrm{~g}$ (Lesson \& Summers 1997, Hernández et al. 2008, Hu et al. 2008).

$\mathrm{Al}$ igual que para humanos, la harina de $L$. olivacea es una buena fuente de ácidos grasos y en relación con su contenido de aminoácidos, se observó que el $40 \%$ de éstos presentes en $L$. olivacea (Met, Cis, Fen, Tir, Tre, Ser), tuvieron una mayor concentración en comparación con las harinas de pescado (Hernández et al. 2008, Hu et al. 2008); además se identificaron dos aminoácidos que no se reportan en estas últimas harinas mencionadas: Cis y Pro. El resto de los aminoácidos se cuantificó en cantidades similares entre las harinas.

Por la calidad y cantidad de los nutrimentos encontrados en la harina de huevos no eclosionados de L. olivacea, se sugiere evaluar su inclusión en la dieta de aves y especies acuícolas. De manera que en los primeros, además de los posibles beneficios en ganancia de peso y conversión alimenticia, el huevo y la carne se podrían enriquecer con EPA y DHA; mientras que en la acuacultura se podría aprovechar para el cultivo de camarón y algunas especies de pescado de alto valor comercial.

En relación con el manejo y conservación actual de L. olivacea en México, desde el inicio de los años 90’s se creó el Centro Mexicano de la Tortuga en donde se llevan a cabo las siguientes actividades: 1) realización de investigación científica y tecnológica para el manejo, desarrollo y conservación de la tortuga, 2) difusión del conocimiento de la biología, conservación y legislación para la protección de las tortugas, 3) promoción del concepto de turismo ecológico en la región (difusión y educación ambiental) y 4) apoyo al desarrollo y crecimiento de las comunidades de la región. Para el avance de estas actividades se cuenta con: Área de Investigación, Área de Difusión y Promoción, Área acuarística, Área de sanidad animal, Laboratorio de monitoreo, campamentos tortugueros (CONANP 2010b, CMT 1995).

L. olivacea se presenta en grandes arribazones en Ostional, Costa Rica, lugar donde se ha establecido una explotación racional de 
los huevos a través de una legislación (Wildlife Conservation Law 6919), que aprueba durante las primeras 36 horas de la arribada, la recolección de 100 huevos por familia para su posterior comercialización. Este manejo ha permitido que el número de huevos recolectados, después de la aplicación de esta ley, sea menor en comparación con el número de huevos que se sustraían cuando no se permitía su recolecta, lo cual ha generado una mejor conservación de la especie. Esto ha dado seguridad y beneficios significativos a la población local (Campbell 1998).

El huevo de tortuga marina L. olivacea liofilizado es un ingrediente con presencia importante, en cantidad y tipo, de nutrimentos de interés tanto para la alimentación humana como animal, principalmente aminoácidos, vitaminas y ácidos grasos n-3 (EPA y DHA). $\mathrm{Su}$ aprovechamiento podría darse a través de la inclusión de esta harina en el desarrollo de alimentos funcionales para humanos y como sustituto de fuentes de proteína en alimentos balanceados para animales, con los beneficios complementarios de los nutrimentos antes mencionados. Con lo anterior se plantea una opción de aprovechamiento del desperdicio de nutrimentos, que actualmente existe en lugares donde se llevan a cabo grandes arribazones de L. olivacea como en La Escobilla, Oaxaca, México, para disminuir en cierta medida el impacto ecológico de las mismas.

\section{AGRADECIMIENTOS}

La autora agradece a Federico Páez-Osuna del Instituto de Ciencias del Mar y Limnología, de la Universidad Nacional Autónoma de México, por las muestras de huevo liofilizado y los datos de las tortugas proporcionadas durante la dirección de un trabajo de tesis y obtenidas con el permiso especial DGVSSEMARNAT No. 06495, Sara Montaño del Depto. de Nutrición Animal del INCMNSZ por el apoyo técnico en el laboratorio y a Martha Harfush del Centro Mexicano de la Tortuga por sus comentarios sobre el estado actual de la tortuga golfina en México.

\section{RESUMEN}

Por su potencial como fuente alimenticia, se analizó la composición química de huevo de Lepidochelys olivacea en La Escobilla, Oaxaca, México. El Instituto de Ciencias del Mar y Limnología de la Universidad Nacional Autónoma de México proporcionó 250 muestras de huevo liofilizado de 25 tortugas, que se analizaron siguiendo métodos oficiales para humedad, cenizas, proteína, grasa, lípidos totales(LT) y análisis microbiológicos; además de ácidos grasos(AG) por cromatografía de gases, aminoácidos(AA), vitaminas y colesterol(col) por HPLC. Los resultados fueron los siguientes: $(\mathrm{g} / 100 \mathrm{~g})$ humedad(4.7), cenizas(3.8), proteína(53.7) y grasa(47.4). AA esenciales (g aa/100g Proteína): Ile (4.4), Lys(6.6), Leu(7.4), Met+Cys (8.8), Phe+Tyr (10.8); retinol $(340 \mu \mathrm{g} / 100 \mathrm{~g})$, colecalciferol $(5.9 \mu \mathrm{g} / 100 \mathrm{~g})$, tocoferol (8.6), tiamina (0.3) y riboflavina (1.1) (mg/100g). $\mathrm{Y}$ los de (LT), (AG) y (Col) se concentraron en tres grupos por peso de tortuga: (LT) (44.3-48.7-49.1g/100g), (Col) (518.4-522.5-728.7 mg/100g). Entonces se identificaron 17 AGSaturados, 8 AGMonoinsaturados y 11 AGPoliinsaturados. Los AGS más abundantes $(\mathrm{mg} / 100 \mathrm{~g})$ : C16:0 (485-1263), AGM: C16:1 (456-716), C18:1n-9c (9041754) y AGP: C20:4n-6 (105-217), EPA (48-103) у DHA (97-189). También, existió diferencia significativa (Fisher, $\mathrm{p}<0.05$ ) para (Col), AG totales, AGS, AGM, AGP y AG n-3 (EPA+DHA). No se detectó presencia de agentes microbiológicos. El huevo liofilizado de L. olivacea es una opción para el desarrollo de productos alimenticios por su proteína de alto valor biológico y ácidos grasos n-3.

Palabras clave: Lepidochelys olivacea, huevo liofilizado no-eclosionado, valor nutritivo, alimentación humana, alimentación animal.

\section{REFERENCIAS}

Abreu-Grobois, F.A. 1999. Genética poblacional y filogeografía de las tortugas marinas golfina (Lepidochelys olivacea) y laúd (Dermochelys coriacea) en el Pacífico mexicano. Informe final SNIB-CONABIO proyecto No. G007, México.

Akegbejo, Y. \& E. Fasakin. 2008. Use of rendered animal protein meals as fish meal replacer in the diets of the African catfish, Clarias gariepinus (Burchell, 1822) juveniles. Tropicultura 26: 89-92.

Alvabera, P.E. 2007. Memorias de la reunión nacional sobre conservación de tortugas marinas. Veracruz, México. (también disponible en línea: www.conanp. gob.mx/pdf/memorias_reunión_2007.pdf).

Allen, M.S. 2007. Three millennia of human and sea turtle interactions in remote Oceania. Coral Reef 26: 959-970. 
A.O.A.C. 2003. Official methods of analysis. Association of official analytical chemists. Washington, D.C., EEUU.

Austin, H., E. Decker \& V. Collins. 2008. Fatty acids in Poultry and egg products, p. 127-154. In C. Kuang Chow (ed.). Fatty acids in foods and their health implications. CRC. EEUU.

Bligh, G. \& I. Dyer. 1959. A rapid method of total lipid extraction and purification. Can. J. Biochem. Physiol. 37: 911-917.

Bourges, R., E. Casanueva \& J. Rosado. 2005. Recomendaciones de ingestión de nutrimentos para la población Mexicana. Bases fisiológicas. Tomo 1. Panamericana, México.

Bourges, R., E. Casanueva \& J. Rosado. 2009. Recomendaciones de ingestión de nutrimentos para la población Mexicana. Bases fisiológicas. Tomo 2. Panamericana, México.

Calderon, C., F. Paez \& A. Abreu. 2008. Preliminary results about heavy metals concentration in sea turtle (Lepidochelys olivacea) using eggs and blood from a nesting colony of La Escobilla, Oaxaca, México. $28^{\text {th }}$ Annual symposium on sea turtle biology y conservation. México.

Campbell, M.L. 1998. Use them or loose them? Conservation and the consumptive use of marine turtle egg at Ostional, Costa Rica. Env. Conserv. 25: 305-319.

Carrillo, S., E. López, M. Casas, R. Castillo \& F. PérezGill. 2008. Potential use of seaweeds in the laying hen ration to improve the quality of n-3 fatty acids enriched eggs. J Appl. Phycol. 20: 721-728.

Casas, A.G. 1978. Análisis de la anidación de las Tortugas marinas del género Lepidochelys en México. An. Centro Cienc. Mar y Limnol. Univ. Nal. Aut. México 5: $141-157$

Castro-González, M.I. \& R.F. Pérez-Gil. 2001. Ácidos grasos en sardina de tomate de diferentes zonas pesqueras del Pacífico. Arch. Lat. Nut. 51: 53-69.

Castro-González, M.I. \& D. Miranda-Becerra. 2009. Evaluation of phosphorus, protein and n-3 fatty acids contents in 15 marine fish species identifies the most beneficial to renal patients. J. Renal Nutr. 19: $462-468$

Castro-González, M.I. \& B.D. Miranda. 2010. El pescado en la dieta del paciente renal: relación fósforo:ácidos grasos n-3. Rev. Inv. Clin. 62: 44-53
Castro-González, M.I. 2010. Recursos Marinos, p. 129148. In M.M. Chávez, F. Pérez-Gil, E. Mendoza (eds.). Valor nutritivo de los alimentos de mayor consumo. McGraw-Hill, México.

CMT. 1995. Centro Mexicano de la Tortuga. Conocer... para conservar. Boletín. Instituto Nacional de la Pesca. Mazunte, Oaxaca. México.

CONANP. 2009. Entorno. Se aleja del peligro de extinción la tortuga Golfina. Comisión Nacional de Áreas Naturales Protegidas. México, D.F., México. (Consultado: 11 de agosto 2010, www.conanp.gob.mx/ dcei/entorno_old/notas/not6/hoy0601.htm).

CONANP. 2010a. Ficha de identificación. Comisión Nacional de Áreas Naturales Protegidas. México, D.F., México. (Consultado: 19 agosto 2010, www. conanp.gob.mx/pdf_especies/tortuga_golfina.pdf).

CONANP. 2010b. Centro Mexicano de la Tortuga. Comisión Nacional de Áreas Naturales Protegidas. Mazunte, Oaxaca, México. (Consultado: 22 enero 2010, http://www.conanp.gob.mx/difusion/comunicado. php?id_subcontenido=192).

Craven, K., J. Parson, S. Taylor, C. Belcher \& D. Owens. 2008. The influence of diet in fatty acids in the egg yolk of green sea turtles, Chelonia mydas. J. Comp. Physiol. B 178: 495-500.

Cruz-Suarez, L.E., D. Ricque-Marie, M. Tapia-Salazar \& C. Guajardo-Barbosa. 2000. Uso de harina de kelp (Macrocystis pyrifera) en alimentos para camarón, p. 19-22. In L.E. Cruz-Suarez, D. Ricque-Marie, M. Tapia-Salazar, M.A. Olvera-Novoa \& E. Civera-Cerecedo (eds.) Avances en nutrición acuícola. Memorias V simposium internacional de nutrición acuícola. Mérida, México.

Eckert, K., K. Bjorndal, A. Abreu-Grobois \& M. Donnelly. 2000. Técnicas de investigación y manejo para la conservación de las tortugas marinas. Grupo especialista en tortugas marinas. UICN/CSE Publicación No. 4. México.

Folch, M., M. Less \& H. Sloan. 1957. A simple method for the isolation and purification of total lipids from animal tissues. J. Biol. Chem. 226: 497-509.

Harfush-Meléndez, M. \& M. López-Reyes. 2007. Reunión nacional sobre conservación de tortugas marinas. Veracruz, México. (Consultado: 10 julio 2010, www. conanp.gob.mx/pdf/memorias_reunión_2007.pdf)

Hernández, C., J. Sarmiento, B. González \& I. Abdo de la Parra. 2004. Replacement of fish meal with coextruded wet tuna viscera and corn meal in diets for 
White shrimp (Litopenaeus vannamei). Aquac. Res. 35: 1153-1157.

Hernández, C., M.A. Olvera, K. Aguilar, B. González \& I. Abdo de la Parra. 2008. Partial replacement of fish meal by porcine meat meal in practical diets for Pacific white shrimp (Litopenaeus vannamei). Aquaculture 277: 244-250.

Hu, M., Y. Wang, Q. Wang, M. Zhao, B. Xiong, X. Qian, Y. Zhao \& Z. Luo. 2008. Replacementof fish meal by rendered animal protein ingredients with lysine and methionine supplementation to practical diets for gibel carp, Carassius auratus gibelio. Aquaculture 275: $260-265$.

IFOMA. La harina de pescado en la alimentación animal. Sociedad Nacional de Perú. Pesquería.

(Consultado: 22 abril 2010, http://fis.com/snp/harina. htm).

Keller, H.E. (ed.). 1988. Analytical methods for vitamins and carotenoids in feed. Department of vitamin research and development, Roche, Basel, Suiza.

Lesson, S. \& D. Summers. 1997. Comercial poultry nutrition. University Books, Guelph, Canada.

Márquez, R., A.O. Villanueva, C.S. Peñaflores \& O.D. Rios. 1989. Situación actual y recomendaciones para el manejo de las tortugas marinas de la costa occidental mexicana, en especial la tortuga golfina Lepidochelys olivacea. Cien. Pesq. 91: 83-91.

Márquez, R., C. Peñaflores \& J. Vasconcelos. 1996. Olive Ridley turtles (Lepidochelys olivacea) show signs of recovery at La Escobilla, Oaxaca. Mar. Turt. News 75: 5-7.

Mora-Castro, R., A. Chávez \& S. Herrera. 1997. Composición química del huevo de tortuga Lora (Lepidochelys olivacea) y evaluación de su calidad física y microbiológica durante su almacenamiento. Reviteca 6: $10-18$.

Mrosovsky, N. 2010. The future of Ridley arribadas in Orissa: From triple waste to triple win? Departament of Zoology, University of Toronto, Toronto, Ontario, Canadá. (Consultado: 30 enero 2010, www.iwmc.org/ turtles/011113.pdf.).

Norma Mexicana NMX-F-330-S-1979. Huevo entero deshidratado o en polvo. Dehydrated or powdered whole egg. México. (Consultado: 8 agosto 2009,
www.colpos.mx/bancodenormas/nmexicanas/NMXF-330-S-1979.PDF).

Norma Oficial Mexicana NOM-092-SSA1-1994. Bienes y servicios. Método para la cuenta de bacterias aerobias en placa. México. (Consultado 3 junio 2009, www. salud.gob.mx/unidades/cdi/nom/092ssa14.html).

Norma Oficial Mexicana NOM-111-SSA1-1994. Bienes y servicios. Método para la cuenta de mohos y levaduras en alimentos. México. (Consultado 3 junio 2009, www.salud.gob.mx/unidades/cdi/nom/111ssa14. html).

Norma Oficial Mexicana NOM-110-SSA1-1994. Bienes y servicios. Preparación y dilución de muestras de alimentos para su análisis microbiológico. México. (Consultado: 10 mayo 2009, http://www.salud.gob. $\mathrm{mx} /$ unidades/cdi/nom/110ssa14.html).

Norma Oficial Mexicana NOM-113-SSA1-1994. Bienes y servicios. Método para la cuenta de microorganismos coliformes totales en placa. México. (Consultado: 3 Junio 2009, www.salud.gob.mx/unidades/cdi/ nom/113ssa14.html).

Norma Oficial Mexicana NOM-114-SSA1-1994. Bienes y servicios. Método para la determinación de salmonella en alimentos. México. (Consultado: 3 junio 2009, www.salud.gob.mx/unidades/cdi/ nom/114ssa14.html).

Norma Oficial Mexicana NOM-159-SSA1-1996. Huevo, sus productos y derivados. disposiciones y especificaciones sanitarias. México. (Consultado: 8 julio 2009, www.salud.gob.mx/unidades/cdi/ nom/159ssa16.html).

Ocana, M. 2010. Arribada nesting of Olive Ridley sea turtles (Lepidochelys olivacea) at La Escobilla, Mexico. $\mathrm{Ph} . \mathrm{D}$. Thesis, University of Oregon, Oregon, EEUU.

Rice, R. 1999. Fish. Nutritional value, p. 793-803. In M.J. Sadler (ed.). Encyclopedia of human nutrition. Academic, London, United Kongdom.

Richard, J.D. \& D.A. Hughes. 1972. Some observations of sea turtle nesting activity in Costa Rica. Mar. Biol. 16: 297-309.

Sotelo, A. \& L. González. 2000. Huevo en polvo con bajo contenido de colesterol. Características nutricias y sanitarias del producto. Arch. Lat. Nutr. 50(2). (Consultado: 22 enero 2010, www.alanrevista. org/ediciones/2000-2/huevo_polvo_bajo_contenido_colesterol.asp). 
Souci, S., W. Fachmann \& H. Kraut. 2000. Food composition and nutrition tables. Medpharm Scientific, Sttutgard, Germany.

StatSoft Inc. 2007. STATISTICA (data analysis software system). Version 8.0. www.statsoft.com.

Stephenson, K. \& W. Owens. 1999. A comparison of total lipid content and fatty acid composition of egg yolks from the green turtle, Chelonia mydas, and the redeared slider, Trachemys scripta. $19^{\text {th }}$ Annual sea turtle symposium. South Padre Island, Texas, EEUU.

Umemura, U., A. Koik, H. Iso, T. Sankai, T. Sinamoto, S. Sato, M. Lida, K. Handa \& Y. Komachi. 1993.
Population based comparative study on dietary habits and serum fatty acid compositions. Nippon Eiseigaku Sasshy 48: 939-954.

Vicenzo, M., A. De Leonardis \& M. Vicenzo. 2005. Solid phase extraction-gas-chromatographic method to determine free cholesterol in animal fats. J. Food Comp. Anal. 18: 617-624.

Wardlaw, M., S. Hampl \& A. DiSilvestro. 2004. Perspectivas en nutrición. McGraw Hill, México.

Waters, 1993. Manual $N^{\circ}$ WATO52874. Método Waters AccQ-Tag Chemistry Package, Milipore Corporation. Milford, Massachuset, EEUU. 